\title{
Autoconcepto y consumo de sustancias en la adolescencia
}

\section{Self-concept and drug use in adolescence}

María C. Fuentes; Fernando García;

EnRIQUe Gracia; Marisol LILA
| Universidad de Valencia. España.

Enviar correspondencia a:

María C. Fuentes. Universidad de Valencia.

Facultad de Psicología.

Departamento de Metodología de las Ciencias del

Comportamiento.

Avda. Blasco lbáñez, 21.

46010, Valencia (España).

E-mail: M.Castillo.Fuentes@uv.es

\section{RESUMEN}

Este estudio analiza la relación entre el autoconcepto evaluado con una medida multidimensional, el cuestionario Autoconcepto Forma 5 (AF5), y el consumo de sustancias en los adolescentes. A partir de las respuestas de 632 adolescentes (47,5\% mujeres), entre 12 y 17 años ( $M=14,88$ años, $D T=1,71$ años), los resultados mostraron relaciones negativas entre el autoconcepto familiar, académico y físico, y el consumo de sustancias. Si bien se encontraron aparentes relaciones positivas con la dimensión social del autoconcepto, estas relaciones desaparecieron al controlar el efecto estadístico del sexo y la edad de los adolescentes. Además, se incluyen otros índices de ajuste (psicológico, competencia personal, conducta antisocial y socialización parental) de los adolescentes. Los resultados refuerzan la idea del autoconcepto como un importante correlato del bienestar psicológico y un constructo teórico básico para explicar las conductas ajustadas y adaptativas en la adolescencia. Igualmente, nuestros resultados avalan la necesidad de controlar el posible efecto estadistico de terceras variables (el sexo), lo que permitiria explicar algunos resultados contradictorios en la literatura (la relación positiva entre el autoconcepto social y el consumo de drogas) y evitar, asi, llegar a conclusiones erróneas basadas en relaciones espurias.

Palabras clave: Autoconcepto, análisis factorial confirmatorio multigrupo, adolescencia, ajuste psicosocial, consumo de sustancias. recibido: octubre 2010 aceptado: junio 2011

\section{ABSTRACT}

This study analyzes the relationship between a multidimensional measure of self-concept, the Self-concept Form-5 Questionnaire (AF5), and drug use among adolescents. From the responses of 632 participants (47.5\% females) aged 12 to 17 years ( $M=14.88$ years, $S D=1.71$ years), results showed negative relationships between family, academic and physical self-concept, and drug use. Although a positive relationship between social self-concept and drug use was found, this significant relationship disappeared once the age and sex of adolescents was controlled statistically. Moreover, the study includes other adjustment indicators in adolescence (psychological adjustment, personal competence, antisocial behavior and parenting). Results support the idea of self-concept as an important correlate of psychological well-being and a basic theoretical construct for explaining adjusted and adaptive behaviors in adolescence. Likewise, our results underline the need for statistical control of the effect of a third variable (sex), which could explain some contradictory results reported in the literature (a positive relationship between social self-concept and drug use), so as to avoid reaching conclusions based on spurious relationships.

Keywords: Self-concept, multigroup confirmatory factor analysis, adolescence, psychosocial adjustment, drug use. 


\section{INTRODUCCIÓN}

$\mathrm{E}$ I estudio del autoconcepto y su relación con el bienestar psicosocial ha motivado un amplio número de investigaciones en la literatura científica. El concepto que el individuo tiene de sí mismo como un ser físico, social y espiritual (García y Musitu, 1999), tradicionalmente ha constituido una clave explicativa para el adecuado funcionamiento comportamental, cognitivo, afectivo y social (Shavelson, Hubner y Stanton, 1976). Sin embargo, numerosos estudios encuentran relaciones positivas entre indicadores de desajuste y el autoconcepto en la adolescencia, argumentando que el autoconcepto, o algunas de sus dimensiones, constituyen factores de riesgo para conductas desajustadas como el consumo de drogas. Este trabajo cuestiona si las relaciones positivas entre algunas de las dimensiones del autoconcepto y el consumo de drogas son espurias y, por lo tanto, representan simples artefactos metodológicos asociados con el sexo y la edad.

Desde una perspectiva aplicada, los psicólogos dedicados a la intervención perciben diariamente las dificultades que experimentan sus pacientes en el funcionamiento cotidiano. Por ejemplo, una persona deprimida se autopercibe como incapaz para afrontar las tareas cotidianas (American Psychiatric Association, 2000), una persona adicta a las drogas no puede afrontar el día a día sin su dosis (American Psychiatric Association, 2000). Parece sencillo comprender que cuando una persona experimenta dichas dificultades, la valoración que haga de sí misma sea deficitaria (Pérez, López, Cuesta y Caballero, 2005). Pese a los muy distintos y variados componentes que confluyen en estos diferentes problemas humanos, el bajo autoconcepto aparece como un denominador común de estas conductas desajustadas y como un importante componente a potenciar, aunque no el único, en los diferentes programas de intervención psicológica. Por ejemplo, en la Terapia cognitiva de las drogodependencias señalan que una función de la droga es "reanimar la autoestima de manera química, aunque de una forma falsa y temporal" (Beck, Wright, Newman y Liese, 2007). En esta línea, la relación entre el autoconcepto y el consumo de sustancias es un tema ampliamente estudiado, sobre todo en el periodo de la adolescencia (Calafat, Mantecón, Juan, Adrover, Blay, Hughes et al., 2011; Echeburúa y Corral, 2010; Ledoux, Sizaret, Hassler y Choquet, 2000; Prado y Pantin, 2011), en el que, tradicionalmente, se ha considerado el bajo autoconcepto como un clásico factor de riesgo para el consumo de sustancias (Calafat y Becoña, 2005; Calafat y Monserrat, 2003; Pérez et al., 2005).

No obstante, en la literatura se constata la existencia de bastantes resultados contradictorios al analizar la naturaleza de dicha relación. Numerosos autores sugieren que estas inconsistencias pueden atribuirse a la medición del autoconcepto como un constructo unidimensional o multidimensional (Cava, Murgui y Musitu, 2008; Musitu, Jiménez y Murgui, 2007; Swaim y Wayman, 2004). Sin embargo, es poco factible asumir que estas discrepancias en torno a la relación entre el autoconcepto y el consumo de sustancias se deban, únicamente, al tipo de instrumento utilizado para medir el autoconcepto (Schroeder, Laflin y Weis, 1993), puesto que, al revisar la literatura siguiendo este criterio, los resultados siguen siendo inconsistentes. Si bien, las medidas multidimensionales ofrecen mediciones más sensibles, concretas y ajustadas (García, Musitu y Veiga, 2006; Palacios y Zabala, 2007; Shavelson et al., 1976; Tomás y Oliver, 2004; Wylie, 1979) en comparación con las medidas globales e inespecíficas proporcionadas por los modelos unidimensionales (Braken, 1992; Fuentes, García, Gracia y Lila, 2011; García et al., 2006; García, Musitu, Riquelme y Riquelme, 2011; Shavelson et al., 1976), medidas que distorsionan la verdadera relación entre el autoconcepto y el consumo de sustancias (Swaim y Wayman, 2004).

Así, en los estudios que han utilizado medidas unidimensionales, algunos autores concluyen que un bajo autoconcepto está relacionado con altos niveles de consumo (Kavas, 2009; Scheier, Botvin, Griffin y Díaz, 2000; Zamboaga, Schwartz, Jarvis y Van Tyne, 2009), otros no encuentran relación (García y Carrasco, 2003; Kokkevi, Richardson, Florescu, Kuzman y Stergar, 2007; McGee y Williams, 2000); y otros afirman que un alto autoconcepto está asociado a altos niveles de consumo (Oliva, Parra y Sánchez-Queija, 2008). Con respecto a los estudios que utilizan medidas multidimensionales, los resultados son consistentes en afirmar el papel protector del autoconcepto familiar y académico frente al consumo de sustancias (Cava et al., 2008; Martínez-Lorca y Alonso-Sanz, 2003; Musitu et al., 2007; Wild, Flisher, Bhana y Lombard, 2004). No obstante, los resultados en torno al autoconcepto social no son tan consistentes, y la literatura científica proporciona numerosos resultados contradictorios. Así, mientras algunos estudios concluyen que los componentes sociales del autoconcepto se relacionan inversamente con el consumo de sustancias (Téllez, Cote, Savogal, Martínez y Cruz, 2003), otros cuestionan esta afirmación al encontrar relaciones significativas y positivas (Brendgen, Vitaro, Turgeon, Poulin y Wanner, 2004; Musitu et al., 2007; Musitu y Herrero, 2003; Pastor, Balaguer y García, 2006; Wild et al., 2004), de forma que, aquellos adolescentes con un mejor concepto y valoración de sí mismos en el ámbito de las relaciones sociales, que se consideran más competentes socialmente y que tienen facilidad para hacer amigos, son los que presentan mayor consumo de sustancias (Cava et al., 2008). Las mismas inconsistencias son también extensibles al autoconcepto físico (Moreno, Moreno y Cervelló, 2009; Musitu et al., 2007). El argumento aportado por diversos autores para explicar esta relación positiva se basa en la consideración del consumo de sustancias como una conducta fundamentalmente social durante la adolescencia y que, por tanto, los adolescentes que consumen ciertas sustancias se autoperciben como "seres sociales normales" (Musitu et al., 2007). Otros autores han sugerido que una actitud de evitación de riesgos por parte del adolescente está asociado a una menor incidencia de problemas de conducta, pero que, al mismo tiempo, esta actitud puede llevar a un desarrollo deficitario de algunas áreas como el logro de la identidad personal y el autoconcepto (Oliva et al., 2008). Estos resultados que sugieren que las puntuaciones altas en las dimensiones social y física del autoconcepto constituyen un factor de riesgo para el consumo de sustancias, ponen 
en entredicho el papel del autoconcepto como un constructo básico subyacente relacionado con las conductas ajustadas y adaptativas (Shavelson et al., 1976). Ante todas estas conclusiones es inevitable plantearse algunas cuestiones como, por ejemplo, ¿hay que tener una actitud más abierta ante las conductas de riesgo para tener un autoconcepto más alto?, para evitar problemas con el consumo de sustancias ies necesario considerarse socialmente poco competente y con dificultades para hacer amigos? ¿Tener bajo el autoconcepto social y físico durante parte de la adolescencia y a la vez alto, por ejemplo, el autoconcepto académico? Si se trata de un mismo constructo, aunque sea multidimensional, es difícil integrar teóricamente de manera congruente estas inconsistencias entre los distintos ámbitos del mismo constructo y el consumo de sustancias. No obstante, estos resultados y las conclusiones que se derivan de ellos podrían estar basados en relaciones espurias que estén reflejando el efecto de terceras variables no controladas estadisticamente (Amón, 1979; García, Frías y Pascual, 1999).

De esta forma, el principal objetivo del presente estudio es analizar las relaciones entre una medida multidimensional del autoconcepto, el cuestionario AF5 (Autoconcepto Forma 5, de García y Musitu, 1999) y el consumo de sustancias, con el fin de precisar la naturaleza de las relaciones entre ambas variables y aportar explicaciones a las inconsistencias presentes en la literatura científica sobre esta temática. Además, se incluyen otros índices de ajuste psicosocial (psicológico, competencia personal, conducta antisocial y socialización parental) con el fin de analizar si existen relaciones congruentes entre (2) los distintos índices de ajuste evaluados (de manera que los que impliquen ajuste o desajuste se relacionarán positivamente entre sí, y los índices que impliquen ajuste se relacionarán negativamente con los que impliquen desajuste); (3) las dimensiones del autoconcepto y el resto de índices de ajuste incluidos en el estudio; (4) las dimensiones del autoconcepto y el total de criterios de ajuste evaluados (de forma que las diferentes dimensiones presentarán relaciones más intensas con los criterios próximos de ajuste que con criterios menos relacionados).

\section{MÉTODO}

\section{Participantes y procedimiento}

Se estimó necesario un tamaño de la muestra de 567 observaciones para detectar un tamaño del efecto medianopequeño $(|r|=0,15$, para contrastes de dos colas) fijando $\alpha$ y $\beta$ en 0,05 (Faul, Erdfelder, Buchner y Lang, 2009; García, Pascual, Frías, Van Krunckelsven y Murgui, 2008). Se seleccionaron seis centros educativos por muestreo aleatorio simple del listado completo de una Comunidad Autónoma española (García et al., 1999). Cuatro de ellos estaban ubicados en poblaciones con más de 30.000 habitantes (tres públicos y uno concertado) y el resto en poblaciones meno- res (uno público y uno concertado). Se contactó con los directores de los centros (ninguno negó su participación) y se consiguieron los habituales permisos paternos (3\% de desautorizaciones). El 96\% de los alumnos completaron los instrumentos. Se recogieron en sobres cerrados y anónimos que incluian también una hoja elaborada por el tutor del alumno (de la que previamente se había separado su nombre) con datos personales (sexo, fecha de nacimiento y curso), y la nota media del curso anterior (desde suspenso, 0 , a sobresaliente, 4). La muestra fueron 632 participantes, 300 mujeres $(47,5 \%)$ y 332 hombres (52,5\%), con edades entre los 12 y 17 años $(M=14,58 ; D T=1,71)$. Analizando la sensibilidad de la muestra (Faul et al., 2009) se pueden detectar $(N=632$; $\alpha=\beta=0,05)$ tamaños del efecto ligeramente inferiores a los previstos $(|r|=0,14) \mathrm{y}$, en todo caso, la potencia es un poco mayor a la prevista $(N=632 ; \alpha=0,05 ;|r|=0,15$; entonces, $1-\beta=0,968)$.

\section{Instrumentos}

Escala Multidimensional de Autoconcepto AF5 -Autoconcepto Forma 5- de García y Musitu (1999). Este instrumento está basado en el modelo teórico jerárquico y multidimensional de Shavelson et al. (1976). Está compuesto por 30 ítems que evalúan 5 dimensiones, 6 ítems por dimensión, con una escala de respuesta que oscila de 1 ("Totalmente en desacuerdo") a 99 ("Totalmente de acuerdo"). Las cinco dimensiones son: autoconcepto académico (p. ej., "Hago bien los trabajos escolares"); autoconcepto social (p. ej., "Hago fácilmente amigos"); autoconcepto emocional (p. ej., item invertido, "Muchas cosas me ponen nervioso"); autoconcepto familiar (p. ej., "Mi familia me ayudaría en cualquier tipo de problemas"); y autoconcepto físico (p. ej., "Me gusta como soy físicamente"). Diferentes estudios han comprobado empíricamente su estructura dimensional mediante análisis factoriales exploratorios (García y Musitu, 1999) y confirmatorios (Elousa y López-Jaúregui, 2008; García et al., 2006; Murgui, García, García y García, en prensa; Tomás y Oliver, 2004), no detectándose problemas metodológicos con los ítems negativos (García et al., 2011; Tomás y Oliver, 2004). También la validez de constructo de las cinco dimensiones ha sido ampliamente constatada en numerosos estudios (Garaigordobil, Durá y Pérez, 2005; Martínez y García, 2007, 2008; Martínez, García y Yubero, 2007). Los coeficiente alfa de las escalas fueron: académico, 0,908; social, 0,822; emocional, 0,742; familiar, 0,877; y físico, 0,796.

Escala de Estilos de Socialización Parental en Adolescentes -ESPA29- (Musitu y García, 2001). Está compuesta por 212 items que permiten evaluar el estilo de socialización que caracteriza a cada uno de los padres por separado (106 ítems sobre las prácticas de socialización de la figura paterna y otros 106 ítems paralelos sobre las prácticas de socialización de la figura materna). Los adolescentes valoran, con una escala de respuesta de 1 ("Nunca") a 4 ("Siempre"), la actuación de su padre y de su madre en 29 situaciones representativas de la cultura occidental: 13 hacen referencia a cuando sus conductas se ajustan a las normas familiares (p. ej., "Si respeto los horarios establecidos en mi casa"), en las que 
los adolescentes valoran las prácticas parentales de afecto ("Me muestra cariño") e indiferencia ("Se muestra indiferente"), y 16 referidas a cuando sus conductas son contrarias a dichas normas (p. ej., "Si voy sucio y desastrado"), en las que los adolescentes valoran las prácticas parentales de diálogo ("Habla conmigo"); displicencia ("Le da igual"); coerción verbal ("Me riñe"); coerción física ("Me pega"); y privación ("Me priva de algo"). A partir de estas valoraciones se obtiene una medida para cada padre en cada una de las principales dimensiones del modelo de socialización (Darling y Steinberg, 1993; Maccoby y Martin, 1983): Aceptación/Implicación, con las prácticas de afecto, diálogo, indiferencia y displicencia (en las dos últimas se invierten las puntuaciones al relacionarse de manera inversa con la dimensión) y Severidad/Imposición, con las prácticas parentales de coerción verbal, coerción física y privación. Su estructura factorial se ha confirmado en diferentes estudios (en España: Musitu y García, 2001, 2004; y Brasil: Martínez, García, Musitu y Yubero, 2012) y tiene la ventaja de la ortogonalidad de las dos dimensiones principales (Lim y Lim, 2003; Musitu y García, 2001). El coeficiente alfa de las dos dimensiones para el padre y la madre fueron respectivamente: aceptación/implicación, 0,965, 0,957; y severidad/imposición, 0,929, 0,925.

Cuestionario de Evaluación de la Personalidad -PAQ(Rohner, 1990). Este instrumento permite evaluar el ajuste psicológico a partir de las autopercepciones de los adolescentes a cerca de su personalidad y disposiciones conductuales. Está compuesto por 36 ítems que, además de aportar un índice general de desajuste personal, se distribuyen en 6 dimensiones, 6 ítems por dimensión, con una escala de respuesta que oscila de 1 ("Casi nunca es cierto") a 4 ("Casi siempre es cierto"). Las seis dimensiones son: agresión/hostilidad (p. ej., "Pienso mucho en pelearme y portarme mal"); autoestima negativa (p. ej., "Me decepciono de mí mismo con facilidad"); autoeficacia negativa (p. ej., "Me siento incapaz de hacer las cosas bien hechas"); irresponsividad emocional (p. ej., "Me resulta difícil expresar abiertamente mis sentimientos a la gente que quiero"); inestabilidad emocional (p. ej., "Me pongo de mal genio sin ninguna razón"); y visión negativa del mundo (p. ej., "Me parece que la vida está llena de peligros"). El coeficiente alfa para el índice general de desajuste fue de 0,881; para las subescalas: 0,652, 0,780, $0,717,0,690,0,658$ y 0,785 , respectivamente.

La competencia personal se midió con dos índices: logro académico, obtenido a través de la información ofrecida por el tutor de los alumnos sobre la nota media del curso anterior de cada participante (desde suspenso, 0, a sobresaliente, 4); y competencia social, evaluada con una adaptación de la subescala Adolescent Self-Perception Profile (Harter, 1982). Incluye 7 ítems que evalúan la percepción de los adolescentes sobre sus habilidades en el ámbito de las relaciones sociales con sus iguales. La escala de respuesta es de cuatro alternativas sobre dos polos opuestos como anclaje, escogiendo la que más se ajuste a su percepción (p. ej., "Algunos adolescentes hacen amistades con facilidad, pero a otros les cuesta hacer amigos"). El alfa de Cronbach fue de 0,743.
Los problemas de conducta se midieron con dos índices: consumo de sustancias, medido por la cantidad de tabaco, alcohol, marihuana y otras sustancias que el adolescente había consumido en las últimas semanas (García y Gracia, 2009, 2010; Lamborn, Mounts, Steinberg y Dornbusch, 1991; Steinberg, Lamborn, Darling, Mounts y Dornbusch, 1994). Incluye 4 items con una escala de respuesta de 1 ("Nada") a 4 ("Mucho") (p. ej., "En las últimas semanas ¿has consumido bebidas alcohólicas?"). Conducta antisocial, que incluye la evaluación de dos índices: conducta escolar disruptiva, medida a través de 5 ítems que indicaban gamberradas en el colegio con una escala de respuesta desde 1 ("Nunca") a 3 ("Dos o más veces") (p. ej., "Pegar a alguien o participar en peleas dentro del colegio/instituto"); y delincuencia, con 8 ítems que implicaban hechos pre-delictivos o delictivos con una escala de respuesta igual a la anterior (p. ej., "Estropear coches de desconocidos") (García y Gracia, 2009, 2010). Los coeficientes alfa fueron: 0,731 y 0,816, respectivamente.

\section{Análisis de datos}

El análisis se llevó a cabo con los programas EQS6.1 y SPSS17. Primero se realizó un análisis factorial confirmatorio para comprobar si la estructura pentadimensional y oblicua del AF5, en coherencia con los planteamientos teóricos (García y Musitu, 1999; Shavelson et al., 1976), proporciona un mejor ajuste -del modelo a los datos-que los modelos unidimensional y ortogonal. Algunos estudios previos muestran que la distribución de algunos ítems de este instrumento presentan patrones de respuesta con una alta concentración en las puntuaciones altas, obteniendo una distribución asimétrica negativa $y$, por tanto, una desviación de la normalidad multivariada (García et al., 2006; Tomás y Oliver, 2004). Por ello, se ha utilizado el estadístico Satorra-Bentler Scaled Chi-square $\left(S B-\chi^{2}\right)$ (Satorra y Bentler, 2001), específicamente recomendado para el análisis de datos multivariados que no cumplen los supuestos paramétricos (Curran, West y Finch, 1996). Además, se han calculado otros índices robustos: el de ajuste comparativo (CFI), donde los valores $>0,95$ indican un buen ajuste, y los valores $>0,90$, un ajuste aceptable (Marsh y Hau, 1996); y la raíz media cuadrática del error de aproximación (RMSEA; Hu y Bentler, 1999), con un intervalo de confianza del $90 \%$ en torno a RMSEA, para el que se ha sugerido que valores $<0,05$ constituyen un buen ajuste; valores entre 0,05 y 0,08 indican un ajuste aceptable; valores entre 0,08 y 0,10 indican un ajuste mínimo y; valores $>0,10$ indican un ajuste pobre (Browne y Cudeck, 1992).

Además, se comprobó la equivalencia factorial del AF5 a través del sexo, la edad y el grado de consumo de sustancias de los participantes. A partir del sistema de partición por la mediana (percentil 50), se clasificó a los sujetos como consumidores o no consumidores. Este sistema únicamente asume el supuesto del orden dentro de la muestra analizada (Frick, 1996), de manera que el consumo que refleja una categoría es mayor o menor con respecto al de la otra categoría. Se aplicaron cuatro modelos anidados que incrementaban progresivamente las restricciones igualando parámetros libres: a partir del modelo base donde cada parámetro se estimaba 
libremente para cada grupo, se fijaron, primero, la equivalencia de los pesos factoriales para los grupos, después, la misma estructura de varianzas y covarianzas entre los factores y, por último, los errores de medida. En cada paso, al restringir los parámetros del modelo previo, se liberan grados de libertad y se incrementa ji-cuadrado. Si el $\Delta \chi^{2}(\Delta \mathrm{gl})$ es estadísticamente significativo, se rechaza la hipótesis nula de equivalencia entre los modelos. Sin embargo, Cheung y Rensvold (2002) recomiendan que para examinar la invarianza se pruebe la significación del cambio en $\mathrm{CFI}$, de forma que $|\Delta C F| \mid<0,01$ "indica que la hipótesis nula de equivalencia no debería rechazarse".

Para analizar la relación entre las dimensiones del AF5 y el consumo de sustancias; así como con el resto de criterios de ajuste psicosocial, se aplicaron correlaciones de Pearson (Moreno et al., 2009). Posteriormente, se re-analizaron las mismas correlaciones controlando el efecto estadístico del sexo y de la edad para analizar su posible influencia en dicha relación y descartar que las variaciones en estas variables produzcan resultados espurios. Además, considerando el grado de consumo de sustancias, se realizó un diseño factorial multivariado (MANOVA) con cada conjunto de criterios de ajuste (autoconcepto, ajuste psicológico, competencia personal, conducta antisocial y socialización parental). A continuación se aplicaron los análisis de varianza (ANOVAs) para cada conjunto de criterios en los que previamente se obtuvieron diferencias estadísticamente significativas en el MANOVA. Finalmente, se re-analizaron los mismos análisis de varianza incluyendo como covariantes las variables sexo y edad (ANCOVA).

\section{RESULTADOS}

\section{Estructura factorial}

El análisis preliminar de los ítems indica que el ítem 14 ("Mi familia está decepcionada de mí") presenta asimetría (índice $>2$ ). Éste, al mismo tiempo, muestra el nivel más alto de curtosis $(4,55$, índice $<7)$, indicando problemas de normalidad multivariada (Satorra y Bentler, 2001). Los resultados del análisis factorial confirmatorio (Tabla 1) muestran que el modelo pentafactorial-oblicuo del instrumento presenta un mejor ajuste a los datos frente a los modelos unidimensional y pentadimensional-ortogonal. También se confirma que la estructura factorial del modelo es invariante con respecto a las variables analizadas siendo el decremento en $C F I(\Delta C F I)<0,01$. Se comprueba que, a partir del modelo base $\left(\operatorname{Tr}_{0}\right)$, la saturación factorial de los ítems no difiere entre los grupos $\left(\operatorname{Tr}_{1}, \Delta C F\right)_{\text {sexo }}<0,01 ; \Delta C F I_{\text {edad }}<0,01 ; \Delta C F I_{\text {consumo }}<$ $0,01)$, los factores tienen la misma importancia relativa entre ellos y siguen el mismo patrón relacional $\left(\operatorname{Tr}_{2}, \Delta C F I_{\text {sexo }}<0,01\right.$; $\left.\Delta C F l_{\text {edad }}<0,01 ; \Delta C F l_{\text {consumo }}<0,01\right) y$, por último, los errores de medida no afectan diferencialmente a los grupos $\left(\operatorname{Tr}_{3}, \Delta C \mathrm{CF}_{\text {sexo }}\right.$ $\left.<0,01 ; \Delta C F I_{\text {edad }}<0,01 ; \Delta C F I_{\text {consumo }}<0,01\right)$.

\section{Autoconcepto y criterios de ajuste}

En la tabla 2 se presenta la matriz de correlaciones de las variables utilizadas en el estudio. En cuanto a la relación entre el autoconcepto y el consumo de sustancias, se obtienen

Tabla 1. Análisis factorial confirmatorio del ajuste de los modelos a los datos y multimuestra por sexo, edad y consumo de sustancias

\begin{tabular}{|c|c|c|c|c|c|c|c|}
\hline MODELO & $S B-\chi^{2}$ & gl & $\mathrm{SB}-\chi^{2} / g \mathrm{I}$ & $\Delta S B-\chi^{2}$ & RMSEA [IC 90\%] & $\mathrm{CFI}$ & $\Delta \mathrm{CFI}$ \\
\hline Tr. Teórico+r $r_{\text {error }} \#$ & 1083,05 & 390 & 2,78 & $-315,87$ & $0,05[0,05-0,06]$ & 0,90 & 0,05 \\
\hline T. Teórico: 5 factores oblicuos & 1398,92 & 395 & 3,54 & $-286,41$ & $0,06[0,06-0,07]$ & 0,85 & 0,04 \\
\hline 0.5 factores ortogonales & 1685,33 & 405 & 4,16 & $-2666,18$ & $0,07[0,07-0,07]$ & 0,81 & 0,41 \\
\hline U. Unifactorial & 4351,51 & 405 & 10,74 & & $0,12[0,12-0,12]$ & 0,40 & \\
\hline \multicolumn{8}{|l|}{ Sexo (hombres vs. mujeres) } \\
\hline Tr0. Teórico+r & 1589,62 & 780 & 2,04 & & $0,04[0,04-0,04]$ & 0,88 & \\
\hline Tr1. Igual saturación de los factores & 1641,69 & 805 & 2,04 & 52,07 & $0,04[0,04-0,04]$ & 0,88 & $<0,01$ \\
\hline Tr2. Igual var./covarianza factorial & 1667,81 & 820 & 2,03 & 26,12 & $0,04[0,04-0,04]$ & 0,88 & $<0,01$ \\
\hline Tr3. Igual varianza de errores & 1675,52 & 850 & 1,97 & 7,71 & $0,04[0,04-0,04]$ & 0,88 & $<0,01$ \\
\hline \multicolumn{8}{|l|}{ Edad (12-14 vs. $15-17)$} \\
\hline Tro. Teórico+r \# \#ultimuestra & 1497,41 & 780 & 1,92 & & $0,04[0,04-0,04]$ & 0,89 & \\
\hline Tr1. Iqual saturación de los factores & 1519,58 & 805 & 1,89 & 22,17 & $0,04[0,04-0,04]$ & 0,89 & $<0,01$ \\
\hline Tr2. Igual var./covarianza factorial & 1534,04 & 820 & 1,87 & 14,46 & $0,04[0,04-0,04]$ & 0,89 & $<0,01$ \\
\hline Tr3. Igual varianza de errores & 1544,88 & 850 & 1,82 & 10,84 & $0,04[0,04-0,04]$ & 0,89 & $<0,01$ \\
\hline \multicolumn{8}{|c|}{ Consumo (Consumidor vs. No consumidor) } \\
\hline Tr0. Teórico+r $r_{\text {error }} \#$ multimuestra & 1545,90 & 780 & 1,98 & & $0,04[0,04-0,04]$ & 0,89 & \\
\hline Tr1. Igual saturación de los factores & 1572,11 & 805 & 1,95 & 26,21 & $0,04[0,04-0,04]$ & 0,89 & $<0,01$ \\
\hline Tr2. Igual var./covarianza factorial & 1590,49 & 820 & 1,94 & 18,38 & $0,04[0,04-0,04]$ & 0,88 & $<0,01$ \\
\hline Tr3. Igual varianza de errores & 1682,66 & 850 & 1,92 & 92,72 & $0,04[0,04-0,04]$ & 0,88 & $<0,01$ \\
\hline
\end{tabular}

* IC: Intervalo de confianza del estadístico RMSEA para el 90\%.

\# El modelo Tr es el mismo que el $T$, excepto que en el Trse ha liberado la independencia para los errores en los pares: 16-26, 12-22, 8-28, 4-14 y 10-25. 


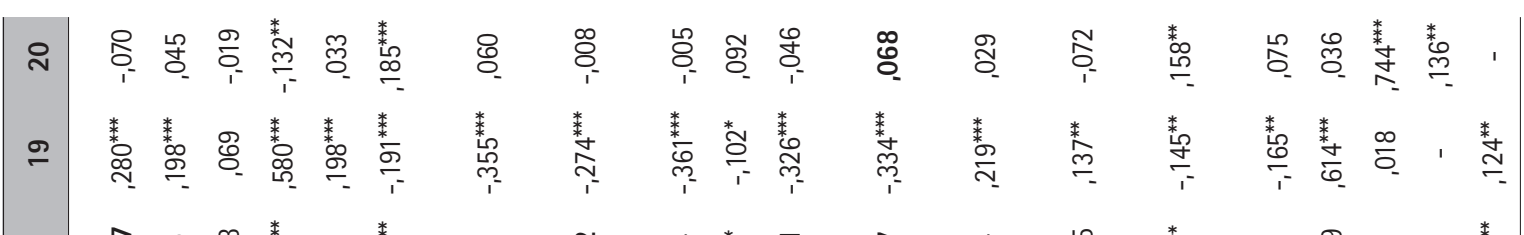

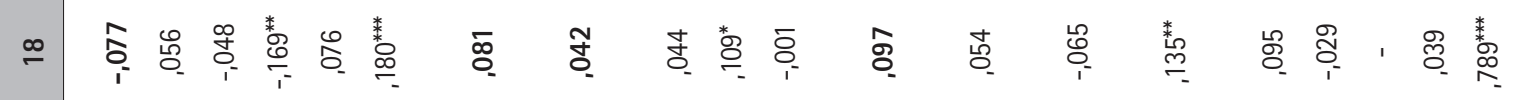

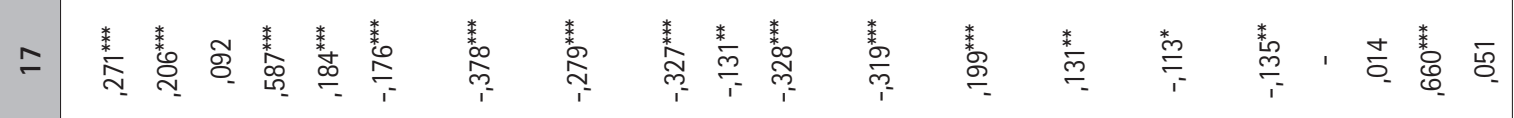

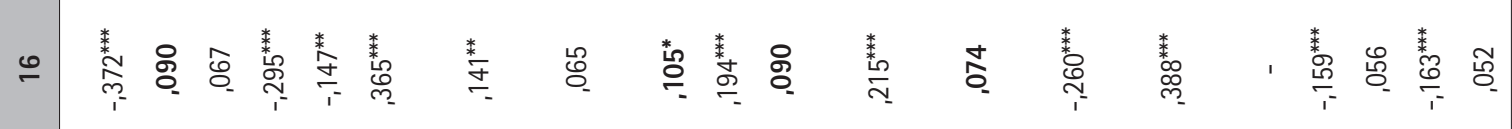

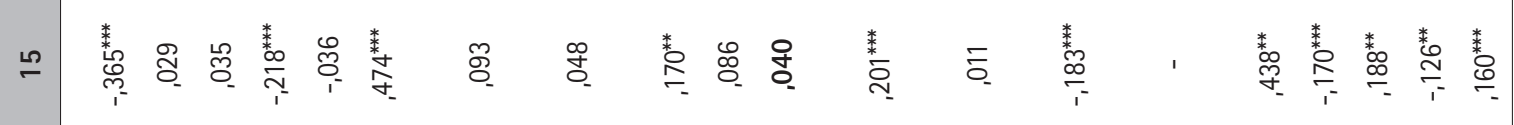

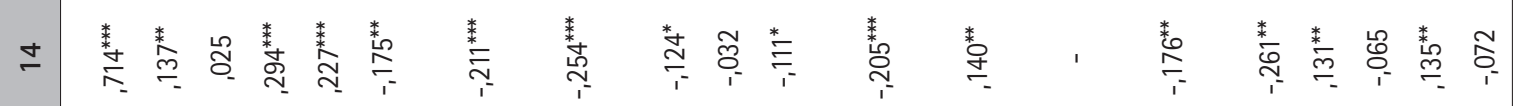

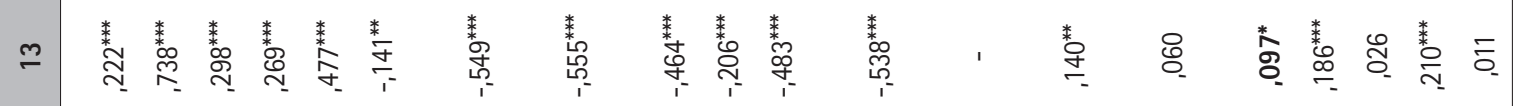

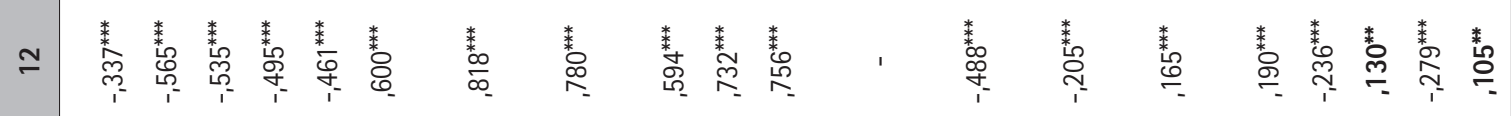

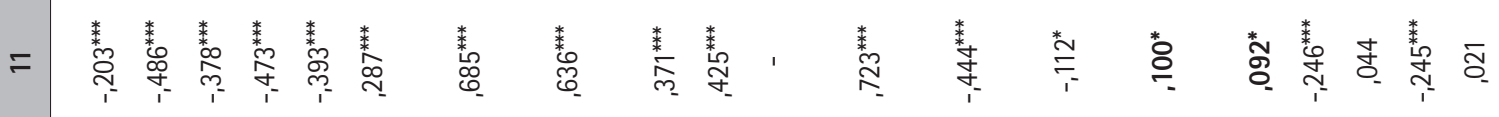

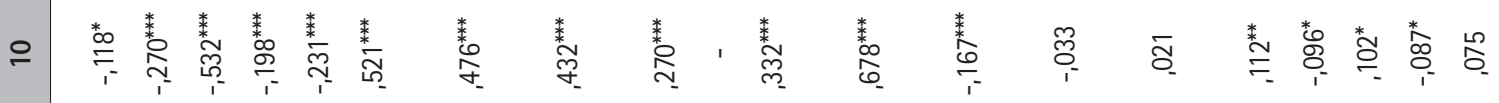

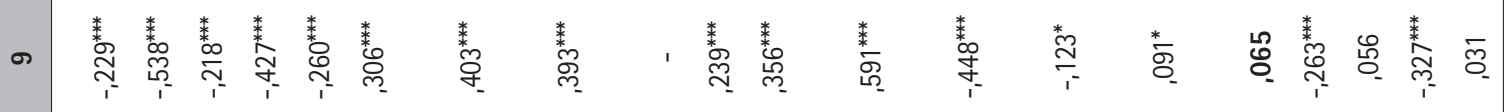

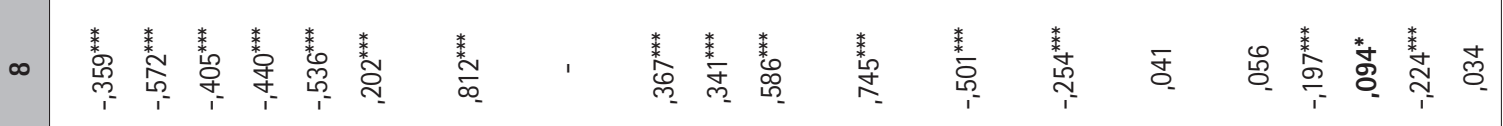

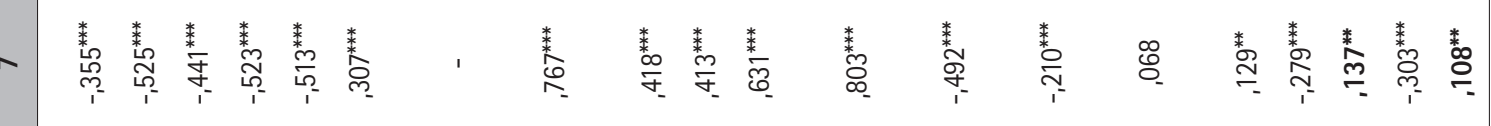

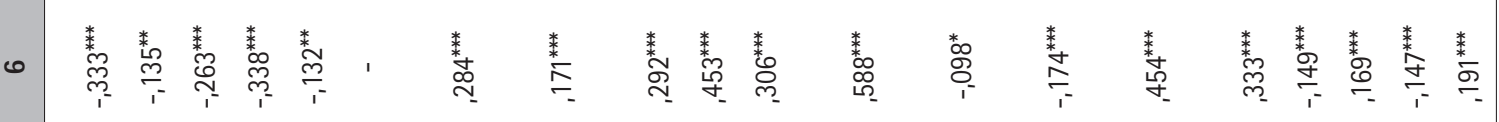

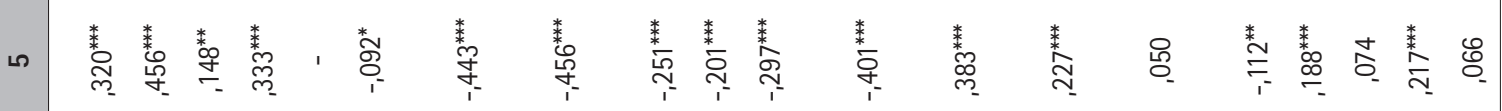

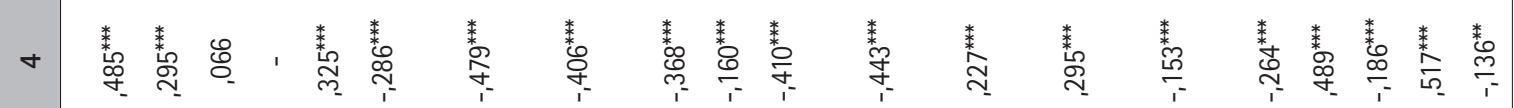

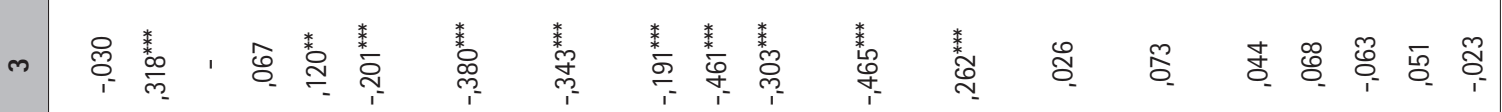

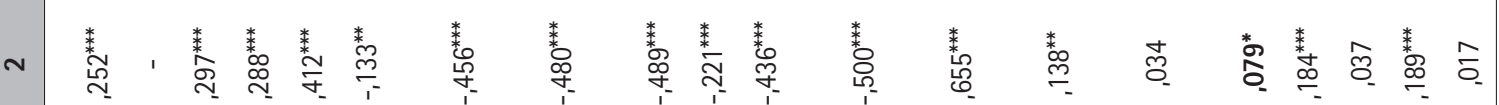

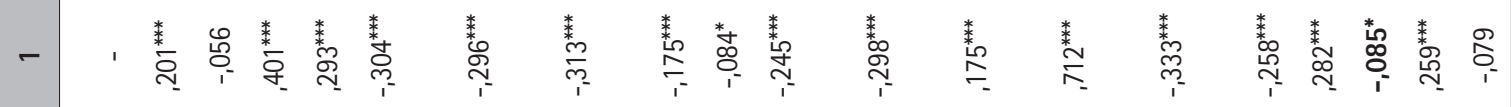


correlaciones significativas y negativas con las dimensiones académica, familiar y física del autoconcepto, relaciones que persisten al aplicar la correlación parcial. El autoconcepto social se relaciona positivamente con el consumo de sustancias. Sin embargo, esta relación deja de ser significativa cuando se aplica la correlación parcial. De forma similar, se observa también una correlación positiva entre consumo de sustancias y competencia social. De nuevo, esta correlación desaparece al aplicar la correlación parcial. Las otras variables que cambian su significación al controlar el efecto estadístico del sexo y la edad lo hacen en el sentido de resultar significativas y en ningún caso implican resultados incoherentes, entre sí ni con el resto de la estructura de resultados.

Con respecto al resto de criterios, los resultados muestran que los índices que implican ajuste o desajuste se relacionan positivamente entre sí (p. ej., agresión/hostilidad y consumo de drogas) y los índices que implican ajuste se relacionan negativamente con los que implican desajuste (p. ej., competencia social e inestabilidad emocional). La relación entre las dimensiones del autoconcepto y el resto de criterios de ajuste incluidos en el estudio son congruentes: se obtienen relaciones positivas con los criterios que implican ajuste (p. ej., autoconcepto físico y competencia social) y negativas con los criterios que implican desajuste (p. ej., autoconcepto académico y desajuste personal). Por último, se observa que las dimensiones del autoconcepto se relacionan más intensamente con los índices de ajuste de los ámbitos más cercanos (p. ej., la relación entre el autoconcepto familiar y la aceptación/implicación de ambos padres es muy superior que su relación con el autoconcepto social, si bien las dos son significativas).

Los MANOVAs aplicados considerando el grado de consumo de sustancias, mostraron diferencias estadísticamente significativas en autoconcepto, $\Lambda=0,950, F(5,615)=$ $6,470, p<0,001$, ajuste psicológico, $\Lambda=0,939, F(7,608)=$ $5,684, p<0,001$, conducta antisocial, $\Lambda=0,944, F(1,620)$ $=36,766, p<0,001$, y socialización parental, $\Lambda=0,983, F(4$, $627)=2,632, p<0,05$. Los resultados de los ANOVAs aplicados individualmente con las variables de estos conjuntos se presentan en la Tabla 3. Las dimensiones académica, familiar y física del autoconcepto mostraron diferencias estadísticamente significativas en función del grado de consumo, siendo los adolescentes no consumidores los que obtuvieron las puntuaciones más altas, relaciones que persistieron al aplicar el ANCOVA. El autoconcepto social también mostró diferencias estadísticamente significativas correspondiendo a los adolescentes consumidores las puntuaciones más altas. No obstante, al aplicar el ANCOVA, con las variables sexo y edad como covariadas, nuevamente la relación entre el autoconcepto social y el grado de consumo de sustancias dejó de ser estadísticamente significativa, $F(1,617)=3,418, p>0,05$.

En cuanto a los ANOVAs realizados con el resto de criterios (ajuste psicológico, conducta antisocial y socialización parental) (Tabla 3), se obtuvieron diferencias estadisticamente significativas correspondiendo a los adolescentes

Tabla 3. Medias, medias ajustadas\#, valores $F$, valores $F$ ajustados\#, significación estadística y significación estadística ajustada\# en función del consumo-no consumo de los participantes

\begin{tabular}{|c|c|c|c|c|c|c|c|c|}
\hline Fuente de variación & $M_{\text {consumo }}$ & $M_{\text {consumo }}^{\#}$ & $M_{\text {no-consumo }}$ & $M_{\text {no-consumo }}^{\#}$ & $F$ & $F^{\#}$ & $p$ & $p^{\#}$ \\
\hline Autoconcepto & & & & & $(1,619)$ & $(1,617)$ & & \\
\hline Autoconcepto Académico & 6,888 & 6,889 & 7,362 & 7,360 & 7,211 & 7,043 & 0,007 & 0,008 \\
\hline Autoconcepto Social & 7,397 & 7,391 & 7,090 & 7,107 & 4,050 & 3,418 & 0,045 & 0,065 \\
\hline Autoconcepto Emocional & 5,754 & 5,754 & 5,758 & 5,757 & 0,001 & $<0,001$ & 0,980 & 0,985 \\
\hline Autoconcepto Familiar & 7,864 & 7,857 & 8,475 & 8,494 & 14,205 & 15,345 & $<0,001$ & $<0,001$ \\
\hline Autoconcepto Físico & 5,555 & 5,537 & 5,928 & 5,975 & 3,928 & 5,447 & 0,048 & 0,020 \\
\hline Ajuste Psicológico & & & & & $(1,614)$ & $(1,612)$ & & \\
\hline Agresión/Hostilidad & 11,642 & 11,641 & 10,485 & 10,489 & 16,643 & 16,280 & $<0,001$ & $<0,001$ \\
\hline Autoestima Negativa & 11,208 & 11,215 & 10,651 & 10,633 & 3,122 & 3,383 & 0,078 & 0,066 \\
\hline Autoeficacia Negativa & 11,311 & 11,324 & 11,379 & 11,344 & 0,053 & 0,005 & 0,817 & 0,944 \\
\hline Irresponsividad Emocional & 13,528 & 13,535 & 13,160 & 13,141 & 1,263 & 1,432 & 0,262 & 0,232 \\
\hline Inestabilidad Emocional & 16,027 & 16,031 & 15,177 & 15,166 & 8,710 & 8,945 & 0,003 & 0,003 \\
\hline Visión Negativa Mundo & 10,705 & 10,709 & 11,154 & 11,144 & 1,985 & 1,845 & 0,159 & 0,175 \\
\hline Desajuste Personal & 91,767 & 91,810 & 88,763 & 88,650 & $\begin{array}{c}5,480 \\
(1,620)\end{array}$ & $\begin{array}{c}6,001 \\
(1,618)\end{array}$ & 0,020 & 0,015 \\
\hline Conducta Antisocial & 1,276 & 1,273 & 1,123 & 1,129 & 36,766 & 33,156 & $<0,001$ & $<0,001$ \\
\hline Socialización Parental & & & & & $(1,630)$ & $(1,628)$ & & \\
\hline Aceptación/Implicación ${ }^{[m a d r e]}$ & 3,178 & 3,180 & 3,301 & 3,295 & 7,851 & 6,732 & 0,005 & 0,010 \\
\hline Severidad/Imposición ${ }^{[\text {madre] }}$ & 1,696 & 1,697 & 1,709 & 1,707 & 0,179 & 0,106 & 0,672 & 0,745 \\
\hline 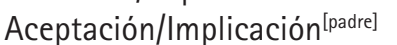 & 2,968 & 2,970 & 3,120 & 3,115 & 9,287 & 8,320 & 0,002 & 0,004 \\
\hline Severidad/Imposición ${ }^{[p a d r e]}$ & 1,633 & 1,633 & 1,655 & 1,654 & 0,517 & 0,408 & 0,472 & 0,523 \\
\hline
\end{tabular}

Nota: En negrita se ha marcado los valores que varían su significación $(\alpha=0,05)$ con los ANCOVAs. 
consumidores las puntuaciones más altas en los criterios que indican desajuste (p. ej., agresión/hostilidad), y a los adolescentes no consumidores, las puntuaciones más altas en los criterios de ajuste (p. ej., aceptación/implicación de ambos padres). Todas estas relaciones persistieron al realizar los ANCOVAs.

\section{DISCUSIÓN}

El presente estudio analiza la relación entre tres conjuntos de variables: una medida multidimensional de autoconcepto -que mide las dimensiones académica, social, emocional, familiar y física-, el consumo de sustancias y otros indicadores del ajuste psicosocial -psicológico, competencia personal, conducta antisocial y socialización parentalde los adolescentes. Los resultados de este trabajo indican que las relaciones positivas entre el consumo de drogas y el autoconcepto social desaparecen una vez se controla el efecto estadístico de terceras variables demográficas (sexo y edad) mediante la correlación parcial (Amón, 1979) o el análisis de covarianza (García et al., 1999). Atendiendo a las discrepancias presentes en la literatura científica a cerca de la naturaleza de las relaciones entre el autoconcepto y el consumo de sustancias, este trabajo pretende contribuir a la búsqueda de explicaciones ante tales inconsistencias (Brendgen et al., 2004; Cava et al., 2008; Moreno et al., 2009; Musitu et al., 2007; Pastor et al., 2006; Wild et al., 2004). Así, los resultados obtenidos en torno a dicha relación indican que (1) las dimensiones académica, familiar y física del autoconcepto se relacionan inversamente con el consumo de sustancias. (2) Los componentes sociales del autoconcepto se relacionan, en un primer momento, de forma positiva con el consumo de sustancias. No obstante, esta relación deja de ser significativa al controlar el efecto estadístico del sexo y la edad, tanto al aplicar las correlaciones parciales como los análisis de covarianza.

Los resultados de este trabajo replican los resultados de otros (Brendgen et al., 2004; Cava et al., 2008; Wild et al., 2004) que han relacionado el consumo de drogas negativamente con varias dimensiones del autoconcepto (las dimensiones académica, familiar y física) y, a la vez, positivamente con otras (la dimensión social). Además, se aprecia que no es difícil reproducir estos resultados porque se ha empleado una medida del consumo muy sencilla que consiste simplemente en dicotomizar la muestra. Esta relación positiva y significativa entre el consumo de drogas y la dimensión social del autoconcepto desaparece al controlar el efecto estadístico de terceras variables (sexo y edad). Resultado que sólo ha sido previamente considerado en un único estudio que estaba limitado al análisis del ajuste general del adolescente y únicamente aplicó la correlación parcial (Fuentes et al., 2011). En este trabajo se extienden los resultados tan limitados analizando el controvertido aspecto de la relación entre el consumo de drogas y la medida multidimensional del autoconcepto durante la adolescencia, constatando las rela- ciones espurias en la relación positiva del consumo de drogas y el autoconcepto tanto al controlar el efecto de las terceras variables con la correlación parcial como con el análisis de la covarianza.

Con respecto al resto de criterios incluidos en el estudio (ajuste psicológico, conducta antisocial y socialización parental), después de controlar el efecto de terceras variables, los resultados muestran relaciones teóricamente congruentes, de forma que, los criterios de ajuste se relacionan de forma positiva entre sí, y negativamente con los que implican desajuste; y las dimensiones del autoconcepto se relacionan positivamente con los indicadores de ajuste y de forma negativa con los criterios de desajuste, mostrándose también relaciones más intensas entre éstas y los criterios más próximos (p. ej., autoconcepto académico y logro académico; autoconcepto emocional e inestabilidad emocional).

Además, los resultados de este trabajo también confirman que el modelo pentafactorial-oblicuo del AF5 se ajusta mejor a los datos que los modelos unidimensional (MartinAlbo, Núñez, Navarro y Grijalvo, 2007; Rosenberg, 1965; Wylie, 1979) y ortogonal, que no tiene en cuenta que las distintas proximidades entre las ramas de la organización jerárquica del autoconcepto produce diferentes grados de relación entre las cinco dimensiones (Shavelson et al., 1976). Estos resultados apoyan los obtenidos en otros estudios sobre la estructura pentafactorial del AF5 (Elousa y LópezJaúregui, 2008; Fuentes et al., 2011; Garcia et al., 2011; Garcia et al., 2006; Murgui et al., en prensa; Tomás y Oliver, 2004). En cuanto al análisis de la invarianza, en este trabajo también se amplian los resultados obtenidos en otros estudios que se han limitado a las variables sexo y edad (Elousa y Muñiz, 2010; García et al., 2011) considerando también el consumo de drogas, aportando que la estructura factorial del instrumento es equivalente entre los diferentes grupos que se comparan (hombres vs. mujeres, 12 - 14 años vs. $15-17$ años, consumidores vs. no consumidores). De esta forma, las diferencias encontradas entre las variables analizadas no se pueden atribuir a las características psicométricas del instrumento (Elousa y Muñiz, 2010).

Es importante destacar que la propia tipificación de los instrumentos que evalúan autoconcepto ya diferencian habitualmente entre sexo y edad, dadas las variaciones que se producen en este constructo según estas variables demográficas (García y Musitu, 1999). Así pues, en esta investigación se plantea, como aportación principal, la necesidad de controlar el efecto de terceras variables en el estudio de las relaciones entre el autoconcepto y el consumo de drogas con el fin de descartar que los resultados obtenidos sean espurios. Un caso semejante de resultados estadísticos mediados por problemas metodológicos, y que también interviene seriamente en la acumulación consistente del conocimiento científico, es el del conocido error del tipo II. Como Cohen $(1962,1994)$ advertía a la comunidad científica, el tamaño de la muestra podía constituir un aspecto tan relevante como el mismo hecho de que las relaciones entre la variable independiente y la dependiente fuesen reales (García et al., 2008; García, Gracia, Fuentes, Lila y Pascual, 2010). De esta 
forma, las repercusiones de este trabajo van dirigidas a que cuando se encuentren las relaciones positivas y negativas se compruebe si éstas no desaparecen al controlar, en las correlaciones o en el análisis de varianza, el efecto de las terceras variables (sexo y edad).

Como en todo trabajo científico, el presente estudio presenta aspectos positivos y limitaciones. Entre los aspectos positivos cabe destacar (1) que se ha analizado uno de los más amplios conjuntos de criterios (20 criterios) para determinar el ajuste psicosocial del adolescente consumidor o no de drogas. (2) Que se ha empleado una muestra con un número de observaciones suficiente para detectar tamaños del efecto pequeños. (3) Que se ha analizado multidimensionalmente el autoconcepto y analizado específicamente que resulta invariante respecto del consumo o no de sustancias, garantizando que el significado de las dimensiones medidas es el mismo para consumidores y no consumidores. (4) Que se ha comprobado mediante dos técnicas estadísticas diferentes el efecto de las terceras variables, para la correlación y el análisis de la varianza. En las limitaciones, realmente la metodología no-experimental de este estudio restringe la validez interna de las relaciones entre las variables estudiadas. No se puede afirmar categóricamente que el ajuste se relacione con las drogas de manera que el inicio en el consumo se deba a un mal ajuste o al revés (Baumeister, Campbell, Krueger y Vohs, 2003). Sin embargo, si que plantea una ventaja importante en relación con la validez externa. Que se pueden analizar simultáneamente un amplio número de criterios, cuando en otro tipo de estudios (cuasiexperimentales y experimentales) aunque la validez interna es mayor, el número de variables analizadas siempre es menor (Cook y Campbell, 1979; Galdós y Sánchez, 2010; Parra y Oliva, 2009; Pérez, 2008; Veiga, García, Neto y Almeida, 2009). La metodología utilizada en este estudio permite observar que hay una relación consistente entre todas las variables analizadas, aspecto que analizando solo consumo y autoconcepto o sus dimensiones no se puede tener en cuenta. Así, se puede observar como, por ejemplo, si no se corrige el efecto de las variables demográficas, a mayor autoconcepto social, mayor consumo de drogas y a la vez mayor relación con la familia (aceptación / implicación). Estas relaciones que analizando por separado cada una de ellas tendrían varias explicaciones, es difícil que se puedan entender al analizar las tres a la vez, como se hace en este estudio. Simplemente, controlando el efecto del sexo y la edad, este resultado desaparece.

Con esta limitación, el presente estudio refuerza los planteamientos teóricos que consideran el autoconcepto como un importante correlato del bienestar psicológico y un constructo básico para explicar las conductas ajustadas y adaptativas (Shavelson et al., 1976). Advirtiendo, al mismo tiempo, de la necesidad de controlar adecuadamente el posible efecto estadístico de terceras variables (como en este estudio, el sexo y la edad) para evitar conclusiones derivadas de relaciones espurias, especialmente si la metodología del diseño no es experimental, y el riesgo de amenaza de la validez interna es más alto (Cook y Campbell, 1979; Pérez, 2008).

\section{AGRADECIMIENTOS}

Este artículo se ha realizado en el marco del Programa VALi+d para investigadores en formación del Plan General Estratégico de Ciencia y Tecnología de la Comunidad Valenciana (2009-2013) (ACIF/2010/282).

\section{REFERENCIAS}

American Psychiatric Association. (2000). Diagnostic and Statistical Manual of Mental Disorders, Text Revision. Washington, D.C.: Autor.

Amón, J. (1979). Estadistica para psicólogos I. Estadistica Descriptiva. Madrid: Pirámide.

Baumeister, R. F., Campbell, J. D., Krueger, J. I. y Vohs, K. D. (2003). Does high self-esteem cause better performance, interpersonal success, happiness, or healthier lifestyles? Psychological Science in the Public Interest, 4, 1-44. doi:10.1111/15291006.01431.

Beck, A. K., Wright, F. D., Newman, C. F. y Liese, B. S. (2007). Cognitive Therapy of Substance Abuse. New York: The Guildford Press.

Bracken, B. A. (1992). Multidimensional self-concept scale examiner's manual. Austin, TX: Pro-Ed Inc.

Brendgen, M., Vitaro, F., Turgeon, L., Poulin, F. y Wanner, B. (2004). Is there a dark side of positive illusions? Overestimation of social competence and subsequent adjustment in aggressive and nonaggressive children. Journal of Abnormal Child Psychology, 32, 305-320. doi:10.1023/B:JACP.0000026144.08470.cd.

Browne, M. W. y Cudeck, R. (1992). Alternative ways of assessing model fit. Sociological Methods and Research, 21, 230-258. doi: $10.1177 / 0049124192021002005$.

Calafat, A. y Becoña, E. (2005). ¿Se puede prevenir el consumo de heroína? Adicciones, 17(Supl. 2), 299-320.

Calafat, A., Mantecón, A., Juan, M., Adrover, D., Blay, N., Hughes, K.,... Rosal, F. (2011). Violent behaviour, drunkenness, drug use and social capital in nightlife. Psychosocial Intervention, 20, 45-51. doi:10.5093/in2011v20n1a4.

Calafat, A. y Monserrat, J. (2003). De la etiología a la prevención del uso y abuso de drogas recreativas. Adicciones, 15(Supl. 2), 261287.

Cava, M. J., Murgui, S. y Musitu, G. (2008). Diferencias en factores de protección del consumo de sustancias en la adolescencia temprana y media. Psicothema, 20,389-395.

Cheung, G. W. y Rensvold, R. B. (2002). Evaluating goodnessof-fit indexes for testing measurement invariance. Structural Equation Modeling, 9, 233-255. doi:10.1207/ S15328007SEM0902_5.

Cohen, J. (1962). Statistical power of abnormal-social psychologicalresearch: A review. Journal of Abnormal Psychology, 65, 145153. doi:10.1037/h0045186. 
Cohen, J. (1994). The earth is round (p-less-than .05). American Psychologist, 49(12), 997-1003. doi:10.1037/0003066X.49.12.997.

Cook, T. D. y Campbell, D. T. (1979). Quasi-experimentation: Design and analysis issues for field settings. Chicago, IL: Rand McNally.

Curran, P. J., West, S. G. y Finch, J. F. (1996). The robustness of test statistics to non-normality and specification error in confirmatory factor analysis. Psychological Methods, 1, 16-29. doi:10.1037/1082-989X.1.1.16.

Darling, N. y Steinberg, L. (1993). Parenting style as context: An integrative model. Psychological Bulletin, 113, 487-496. doi:10.1037/0033-2909.113.3.487.

Echeburúa, E. y Corral, P. (2010). Adicción a las nuevas tecnologías y a las redes sociales en jóvenes: Un nuevo reto. Adicciones, 22, 91-96.

Elosua, P. y López-Jáuregui, A. (2008). Adapting the AF5 autoconcept scale to Basque: Validity evidences. Individual Differences Research, 6, 57-70.

Elosua, P. y Muñiz, J. (2010). Exploring the factorial structure of the self-concept: A sequential approach using CFA, MIMIC, and MACS models, across gender and two languages. European Psychologist, 15, 58-67. doi:10.1027/1016-9040/a000006.

Faul, F., Erdfelder, E., Buchner, A. y Lang, A. G. (2009). Statistical power analyses using $\mathrm{G}^{*}$ Power 3 : Tests for correlation and regression analyses. Behavior Research Methods, 41, 1149-1160. doi:10.3758/BRM.41.4.1149.

Frick, R. W. (1996). The appropriate use of null hypothesis testing. Psychological Methods, 1, 379-390. doi: 10.1037/1082989X.1.4.379.

Fuentes, M. C., Garcia, F., Gracia, E. y Lila, M. (2011). Autoconcepto y ajuste psicosocial en la adolescencia. Psicothema, 23, 7-12.

Galdós, J. S. y Sánchez, I. M. (2010). Relación del tratamiento por dependencia de la cocaína con los valores personales de apertura al cambio y conservación. Adicciones, 22, 51-58.

Garaigordobil, M., Durá, A. y Pérez, J. I. (2005). Psychopathological symptoms, behavioural problems, and self-concept/selfesteem: A study of adolescents aged 14 to 17 years old. Annuary of Clinical and Health Psychology, 1, 53-63.

Garcia, F. y Gracia, E. (2009). Is always authoritative the optimum parenting style? Evidence from Spanish families. Adolescence, 44(173), 101-131.

García, F. y Gracia, E. (2010) ¿Qué estilo de socialización parental es el idóneo en España? Un estudio con niños y adolescentes de 10 a 14 años. Infancia y Aprendizaje, 33, 365-384. doi:10.1174/021037010792215118.

Garcia, F. y Musitu, G. (1999). AF5: Autoconcepto Forma 5. Madrid: Tea.

Garcia, J. F., Frias, M. D. y Pascual, J. (1999). Los diseños de la investigación experimental: Comprobación de las hipótesis. Valencia: Cristóbal Serrano Villalba.

Garcia, J. F., Gracia, E., Fuentes, M. C., Lila, M. y Pascual, J. (2010). La innovación educativa desde la metodología: Mejora de las actitudes y competencias científicas de los alumnos [Educational innovation in research methodology: Improving students' scientific skills and attitudes]. Escritos de Psicología, 3, 1-10. doi:10.5231/psy.writ.2010.0909.

Garcia, J. F., Musitu, G., Riquelme, E. y Riquelme, P. (2011). A confirmatory factor analysis of the "Autoconcepto Forma 5" questionnaire in young adults from Spain and Chile. Spanish Journal of Psychology, 14.

García, J. F., Musitu, G. y Veiga, F. (2006). Autoconcepto en adultos de España y Portugal. Psicothema, 18, 551-556.

García, J. F., Pascual, J., Frias, M. D., Van Krunckelsven, D. y Murgui, S. (2008). Diseño y análisis de la potencia: $n$ y los intervalos de confianza de las medias. Psicothema, 20,933-938.

García, M. A. y Carrasco, A. M. (2003). Factores individuales, familiares y educativos asociados al consumo de alcohol en jóvenes. Revista de Psicología Social, 18, 49-60. doi:10.1174/02134740360521778.

Harter, S. (1982). The perceived competence scale for children. Child Development, 53, 87-97. doi:10.2307/1129640.

Hu, L. y Bentler, P. M. (1999). Cutoff criteria for fit indexes in covariance structure analysis: Conventional criteria versus new alternatives. Structural Equation Modeling: A Multidisciplinary Journal, 6, 1-55. doi:10.1080/10705519909540118.

Kavas, A. B. (2009). Self-esteem and health-risk behaviours among Turkish late adolescents. Adolescence, 44(173), 187-198.

Kokkevi, A., Richardson, C., Florescu, S., Kuzman, M. y Stergar, E. (2007). Psychosocial correlates of substance use in adolescence: A cross-national study in six European countries. Drug and Alcohol Dependence, 86, 67-74. Doi: 10.1016/j. drugalcdep.2006.05.018.

Lamborn, S. D., Mounts, N. S., Steinberg, L. y Dornbusch, S. M. (1991). Patterns of competence and adjustment among adolescents from authoritative, authoritarian, indulgent, and neglectful families. Child Development, 62, 1049-1065. doi:10.2307/1131151.

Ledoux, S., Sizaret, A., Hassler, C. y Choquet, M. (2000). Consumo de sustancias psicoactivas en la adolescencia. Análisis de los estudios de cohorte. Adicciones, 12, 255-279.

Lim, S. L. y Lim, B. K. (2003). Parenting style and child outcomes in Chinese and immigrant Chinese families-current findings and cross-cultural considerations in conceptualization and research. Marriage and Family Review, 35, 21-43.

Maccoby, E. E. y Martin, J. A. (1983). Socialization in the context of the family: Parent -child interaction. En P. H. Mussen (Ed.), Handbook of child psychology (Vol. 4, pp. 1-101). New York: Wiley.

Marsh, H. W. y Hau, K. T. (1996). Assessing goodness of fit: Is parsimony always desirable? Journal of Experimental Education, 64,364-390.

Martín-Albo, J., Núñez, J. L., Navarro, J. G. y Grijalvo, F. (2007). The Rosenberg Self-Esteem Scale: Translation and validation in university students. Spanish Journal of Psychology, 10, 458467. 
Martínez-Lorca, M. y Alonso-Sanz, C. (2003). Búsqueda de sensaciones, autoconcepto, asertividad y consumo de drogas ¿existe relación? Adicciones, 15, 145-158.

Martinez, I. y García, J. F. (2007). Impact of parenting styles on adolescents' self-esteem and internalization of values in Spain. Spanish Journal of Psychology, 10, 338-348.

Martínez, I. y Garcia, J. F. (2008). Internalization of values and self-esteem among Brazilian teenagers from authoritative, indulgent, authoritarian, and neglectful homes. Adolescence, 43(169), 13-29.

Martínez, I., Garcia, J. F., Musitu G. y Yubero, S. (2012). Las prácticas de socialización familiar: confirmación factorial de la versión portuguesa de una escala para su medida [Family socialization practices: Factor confirmation of the Portuguese version of a scale for their measurement]. Revista de Psicodidactica.

Martínez, I., García, J. F. y Yubero, S. (2007). Parenting styles and adolescents' self-esteem in Brazil. Psychological Reports, 100, 731-745. doi:10.2466/PR0.100.3.731-745.

McGee, R. y Williams, S. (2000). Does low self-esteem predict health compromising behaviours among adolescents? Journal of Adolescence, 23, 569-582. doi:10.1006/jado.2000.0344.

Moreno, J. A., Moreno, R. y Cervelló, E. (2009). Relación del autoconcepto físico con las conductas de consumo de alcohol y tabaco en adolescentes. Adicciones, 21, 147-154.

Murgui, S., García, C., García, A. y García, J. F. (en prensa). Autoconcepto en jóvenes practicantes de danza y no practicantes: Análisis factorial confirmatorio de la escala AF5 [Self-concept in young dancers and non-practitioners: Confirmatory factorial analysis of the AF5 Scale]. Revista de Psicología del Deporte.

Musitu, G. y Garcia, F. (2001). ESPA29: Escala de estilos de socialización parental en la adolescencia [ESPA29: Parental socialization scale in adolescence]. Madrid: Tea.

Musitu, G. y Garcia, F. (2004). ESPA29: Escala de estilos de socialización parental en la adolescencia [ESPA29: Parental socialization scale in adolescence] (2nd ed.). Madrid: Tea.

Musitu, G. y Herrero, J. (2003). El rol de la autoestima en el consumo moderado de drogas en la adolescencia. Revista Internacional de Ciencias Sociales y Humanidades, 13, 285-306.

Musitu, G., Jiménez, T. I. y Murgui, S. (2007). Funcionamiento familiar, autoestima y consumo de sustancias: Un modelo de mediación. Revista de Salud Pública de México, 49, 3-10. doi:10.1590/ S0036-36342007000100002.

Oliva, A., Parra, Á. y Sánchez-Queija, I. (2008). Consumo de sustancias durante la adolescencia: Trayectorias evolutivas y consecuencias para el ajuste psicológico. International Journal of Clinical and Health Psychology, 8, 153-169.

Palacios, E. G. y Zabala, A. F. (2007). Los dominios social y personal del autoconcepto. Revista de Psicodidáctica, 12, 179-194.

Parra, A. y Oliva, A. (2009). A longitudinal research on the development of emotional autonomy during adolescence. Spanish Journal of Psychology, 12, 66-75.
Pastor, Y., Balaguer, I. y García, M. (2006). Relaciones entre el autoconcepto y el estilo de vida saludable en la adolescencia media: Un modelo exploratorio. Psicothema, 18, 18-24.

Pérez, J. F. G. (2008). Métodos de investigación, diseño y técnicas en las ciencias del comportamiento. Valencia: Palmero Ediciones.

Pérez, E. J. P., López, M. P., Cuesta, S. E. y Caballero, C. G. (2005). Validación del Cuestionario de Variables de Interacción Psicosocial (VIP): Hacia un modelo de tratamiento de las conductas adictivas "guiado por la personalidad" [Validation of the Psychosocial Interaction Variables Questionnaire: Towards a upersonality guided therapy" model of addiction treatment]. Trastornos Adictivos, 7, 166-186.

Prado, G. y Pantin, H. (2011). Reducing substance use and HIV health disparities among Hispanic youth in the U.S.A.: The Familias Unidas program of research. Psychosocial Intervention, 20, 63-73. doi:10.5093/in2011v20n1a6.

Rohner, R. P. (1990). Handbook for the study of parental acceptance and rejection (3rd Ed.). Storrs, CT: Rohner Research Publications.

Rosenberg, M. (1965). Society and the adolescent self-image. Princeton, NJ: Princeton University Press.

Satorra, A. y Bentler, P. M. (2001). A scaled difference chi-square test statistic for moment structure analysis. Psychometrika, 66, 507514. doi:10.1007/BF02296192.

Scheier, L. M., Botvin, G. J., Griffin, K. W. y Diaz, T. (2000). Dynamic growth models of self-esteem and adolescent alcohol use. Journal of Early Adolescence, 20, 178-209. doi:10.1177/027243 1600020002004.

Schroeder, D. S., Laflin, M. T. y Weis, D. L. (1993). Is there a relationship between self-esteem and drug use? Methodological and statistical limitations of the research. The Journal of Drug Issues, 23, 645-665.

Shavelson, R. J., Hubner, J. J. y Stanton, G. C. (1976). Self-concept: Validation of construct interpretations. Review of Educational Research, 46, 407-441. doi:10.3102/00346543046003407.

Steinberg, L., Lamborn, S. D., Darling, N., Mounts, N. S. y Dornbusch, S. M. (1994). Over-Time changes in adjustment and competence among adolescents from authoritative, authoritarian, indulgent, and neglectful families. Child Development, 65, 754770. doi:10.2307/1131416.

Swaim, R. C. y Wayman, J. C. (2004). Multidimensional self-esteem and alcohol use among Mexican American and white non-latino adolescents: Concurrent and prospective effects. American Journal of Orthopsychiatry, 74, 559-570. doi:10.1037/00029432.74.4.559.

Téllez, J., Cote, M., Savogal, F., Martínez, E. y Cruz, U. (2003). Identificación de factores protectores en el uso de sustancias psicoactivas en estudiantes universitarios. Medicina, 51, 15-24.

Tomás, J. M. y Oliver, A. (2004). Análisis psicométrico confirmatorio de una medida multidimensional del autoconcepto en español. Revista Interamericana de Psicología, 38, 285-293.

Veiga, F., Garcia, F., Neto, F. y Almeida, L. (2009). The differentiation and promotion of students' rights in 
Portugal. School Psychology International, 30, 421-436. doi:10.1177/0143034309106947.

Wild, L. G., Flisher, A. J., Bhana, A. y Lombard, C. (2004). Associations among adolescent risk behaviors and self-esteem in six domains. Journal of Child Psychology and Psychiatry, 48, 14541467. doi:10.1111/j.1469-7610.2004.00330.x.
Wylie, R. C. (1979). The self-concept: Theory and research on selected topics. Lincoln: University of Nebraska Press.

Zamboaga, B. L., Schwartz, S. J., Jarvis, L. H. y Van Tyne, K. (2009). Acculturation and substance use among Hispanic early adolescents: Investigating the mediating roles of acculturative stress and self-esteem. The Journal of Primary Prevention, 30, 315-333. doi:10.1007/s10935-009-0182-z. 